\title{
Une méthode d'analyse des problèmes acoustiques externes et internes en aéronautique. Illustrations dans le cas du véhicule spatial Hermes
}

\author{
F. DUBOIS et P. LAMARY* \\ Dassault Aviation, Centre Spatial Dassault, 17 avenue Didier Daurat, BP. 23, 31701 Blagnac, France \\ * Dassault Aviation, 78 Quai Marcel Dassault, 92214 Saint Cloud, France
}

\begin{abstract}
To solve aeronautical and aerospace industrial vibro-acoustic problems (cabin noise, panel radiation, vibration environment,...) Dassault Aviation has developed an attractive computation strategy. Actually, in the so-called 'Coupling Finite Element and Singularity method' (CFES), each field (structure dynamics, interior acoustics, exterior acoustics) is calculated independently from one another with the best suitable methods. As there is no reason to have coincidence between these optimized subsystems, elasto-acoustic couplings are performed via an original technique based upon the introduction of interfacing models both independent of structure and acoustic approximations. The effectiveness of this approach is fully illustrated through the studies undertaken for Hermes spacecraft project. Severe acoustic loadings were identified (engines noise at launch, take off transonic aeronoise, phases of maximum dynamic pressure during atmospheric reentry ) which may have caused damage to the 'nose' of this glider. Thus, taking profit on our computational organisation, numerical simulations were done considering a mechanical model (of the nose) coupled with the inside acoustic cavity. This made possible numerous sensitivity analyses from the study of correlation length effect to the determination of equivalent loads for shaker tests on a scaled-down model.
\end{abstract}

\section{PRESENTATION DE L'OUTIL DE CALCUL DEVELOPPE PAR DASSAULT AVIATION}

L'outil de calcul développé par Dassault Aviation est un outil d'analyse vibro-acoustique permettant de résoudre les problèmes industriels de couplage d'une structure avec un fluide extérieur ou intérieur.

Il utilise un modèle élastique par Eléments Finis conventionnels de la structure, un modèle par Eléments Finis des fluides internes (basés sur une formulation variationnelle de l'équation d'Helmholtz) et un modèle frontière du fluide extérieur pour la détermination du champ de pression acoustique sur cette frontière (méthode de singularités).

Le principe de calcul consiste à effectuer dans un premier temps les opérations "lourdes" sur chacun des modèles- le calcul des modes propres de la structure (considérée découplée des modes acoustiques) - le calcul des modes propres de la cavité (considérée découplée des modes propres de la structure) - le calcul du champ de pression acoustique externe (fluide au repos ou en 
mouvement). Ce dernier calcul s'effectue fréquence à fréquence mais sur une base réduite de déformées frontière.

Les couplages entre les différents modèles réduits sont ensuite réalisés par l'introduction d'un maillage intermédiaire sur lequel sont calculés des opérateurs gérant en particulier la non coïncidence des finesses de maillage des différents modèles et contenant les seules données intervenant dans le couplage élasto-acoustique. Les opérateurs vérifient au mieux le principe des travaux virtuels aux interfaces de couplage.

L'assemblage de ces équations et opérateurs réduits conduit à l'étude de la stabilité du système fluide/structure couplé, le suivi des différentes variables et paramètres d'analyses.

On trouvera de plus amples détails sur ce procédé de calcul dans les références [1] et [2].

Son intérêt majeur est de permettre d'employer des maillages parfaitement adaptés et optimisés à chacune des entités considérées (structure, acoustique interne et externe), ce qui conduit à une réduction considérable du nombre de degrés de liberté des models et du temps CPU nécessaire aux calculs. Il est à noter que par notre technique le couplage est indépendant des différents cas de répartition des masses structurales. Un des autres avantages de l'outil de calcul développé par Dassault Aviation est d'être intégré aux logiciels CATIA et ELFINI. Il bénéficie ainsi de toutes les fonctionalités logiciels de notre environnement, (mailleur, post-traitement des résultats) et de l'expérience et du savoir faire de Dassault Aviation dans ce domaine.

\section{UNE APPLICATION DE LA METHODE. DEFINITION D'ESSAIS DYNAMIQUES D'UNE PIECE DE DEVELOPPEMENT DE LA POINTE AVANT D'HERMES.}

\subsection{Présentation générale}

Les études de faisabilité de la pointe avant d'Hermes ont conduit à lancer une campagne d'essais visant à soumettre une pièce de développement à l'équivalent d'une vie avion. Cette pièce de développement correspond au modèle de vol tel qu'il a été défini à l'issue des études de faisabilité réduit d'un facteur 0.6. La simulation d'une vie avion est réalisée par soumission de la pièce de développement à des charges d'essais successives statiques, thermiques et dynamiques, les charges d'essais dynamiques étant réalisées sur pot vibrant par raison d'économie et de simplicité. Le rôle attribué aux essais dynamiques dans le cadre de cette simulation est de reproduire un vieillissement de la structure similaire à celui susceptible d'être obtenu à l'issue des différents régimes de sollicitations dynamiques de vol constitutifs d'une vie avion.

Pour définir les spectres d'excitation du pot vibrant destinés à remplir ce rôle, la démarche suivante a été retenue:

1- Estimation des charges dynamiques susceptibles de s'exercer sur la pointe avant au cours d'un vol

2- Identification au moyen de calculs par éléments finis des zones de la pointe avant les plus sollicitées par les différents régimes d'excitation précédemment identifiés et quantification de leurs réponses

3- détermination de spectres de vibration aléatoire qui conduisent, d'après des calculs par éléments finis sur la pièce de développement, à des réponses de cette pièce de sévérité analogue à celle estimée sur le modèle de vol

La synthèse complète de ces travaux figure dans la référence [3]. 


\subsection{Estimation des charges dynamiques de vol s'exerçant sur la pointe avant d'Hermes et des réponses induites dans la structure}

Au cours d'un vol, trois régimes principaux d'excitation dynamique sollicitent le véhicule spatial Hermes : le bruit des moteurs sur le pas de tir et les bruits de couche limite lors de l'obtention des pressions dynamiques maximales au cours du lancement d'une part et de la rentrée d'autre part. La connaissance de ces bruits se résume à des estimations des densités spectrales de puissance et à un bruit sur le pas de tir décrit comme étant de type bruit diffus. La corrélation des pressions des bruits de couche limite est en particulier indéfinie.

Nous avons donc dans un premier temps effectué des calculs par éléments finis sur la pointe avant d'Hermes pour étudier l'influence potentielle de la corrélation des pressions.

Les résultats obtenus montrent qu'une corrélation des pressions de type bruit diffus pour les bruits de couche limite engendre des niveaux de contraintes dans les zones les plus sollicitées six fois plus importants que pour une longueur de corrélation de $200 \mathrm{~mm}$ considérée indépendante de la fréquence et isotrope, ce qui situe le degré d'incertitude engendré par la méconnaissance des corrélations dans les bruits de couche limite.

On constate en outre que les niveaux de contraintes dans les zones les plus sollicitées sont d'autant plus importants que l'interspectre de pression du bruit de couche limite s'harmonise avec la déformée d'un des premiers modes de la structure.

Ceci nous a amené à considérer le bruit diffus comme la référence des excitations acoustiques à considérer lors des régimes d'excitations dynamiques se manifestant lors du lancement et de la rentrée pour être raisonnablement conservatif vis à vis des sollicitations à attendre en vol.

Suite à ce choix nous avons étudié et quantifié les réponses de la pointe avant à la combinaison des excitations acoustiques considérées de type bruit diffus avec les excitations vibratoires estimées transmises par le reste de la structure de l'avion spatial en réponse aux excitations acoustiques de tout l'avion ainsi qu'avec les excitations vibratoires provenant du fonctionnement des moteurs. Pour ce faire, nous avons considéré la structure avec et sans couplage avec sa cavité interne afin de caractériser l'influence d'une cavité dont les premiers modes propres sont situés dans la même plage de fréquence que les premiers modes propres structuraux de la pointe avant.

Il en ressort que les contraintes dans les zones les plus sollicitées de la pointe avant sont augmentées de 5 à $15 \%$ par suite du couplage.

\subsection{Definition des essais dynamiques de la pièce de développement}

Pour définir les charges d'essai, nous avons dans un premier temps étudié les réponses du modèle de la pièce de développement de la pointe avant à une excitation de spectre bruit blanc de niveau forfaitaire selon chacun des trois axes principaux de la pièce.

La comparaison de ces réponses avec celles précédemment obtenues pour le modèle avion montre que les zones les plus sollicitées sont identiques pour les deux modèles.

Cependant, aucune des trois excitations considérées pour la pièce de développement ne permet de représenter fidelement les répartitions d'efforts et de contraintes obtenues dans les zones les plus sollicitées de la pièce avion.

Ceci s'explique par le fait que les efforts et contraintes internes unitaires relatifs aux principaux modes excités, dont la géométrie est pourtant similaire à celle des principaux modes excités du modèle avion, sont sensiblement différents, en raison du facteur d'échelle et de différences locales de géométrie dans les zones de contraintes maximales.

Ceci nous a amené à penser qu'aucune excitation simple (même de type créneaux par exemple) ne permettrait véritablement d'aboutir à la représentation fidèle des niveaux de contraintes dans les zones les plus sollicitées.

Nous avons en conséquence décidé de nous limiter à des charges d'essai de spectre de type bruit blanc en faisant un compromis permettant de solliciter les différentes zones identifiées comme les 
plus sollicitées à un niveau le plus proche possible du niveau maximal estimé en vol sans pour autant générer des niveaux de sollicitation plus critiques.

Ceci nous a conduit à décider de soumettre la pièce à trois excitations successives selon chacun des axes principaux lors de chacun des essais dynamiques prévus dans la campagne, chacune ayant un niveau déterminé afin d'obtenir la même valeur de marge minimale que sur la pièce avion.

La durée prise pour chacune des excitations correspond à la durée d'exposition au niveau maximal d'une zone au cour d'une vie avion (soit au cumul des durées estimées des excitations dynamiques de décollage et de lancement à leur niveau maximal) divisée par le nombre d'essais.

Il convient également de noter que la démarche présentée ici est celle ayant permis de définir les spectres d'excitation à basse fréquence (de 20 à $400 \mathrm{~Hz}$ ) compte tenu des limites de représentativité en fréquence des modèles par éléments finis utilisés.

Pour étendre ces spectres aux fréquence plus élevées, nous avons considéré les spécifications applicables à la pièce avion en admettant de ne pas corriger les niveaux correspondants pour tenir compte de différences d'excitabilité, à haute fréquence, de la pièce avion et de celle de développement.

\section{REFERENCES}

[ 1 ]: ELFINI Vibro-Acoustics-Presentation Manual - DASSAULT AVIATION - 1991

[ 2 ]: Une méthode de couplage Elasto-Acoustique par opérateur de grille frontière pour discrétisation acoustique et structure non coïncidentes - P.LAMARY - Thèse de l'Université de Compiègne - Décembre 1992

[ 3 ]: Détermination et justification des charges d'essais vibratoires pour le nez Echelle 0.6 F.DUBOIS - DASSAULT AVIATION CSD/BC/H1355 - marché 91/ESA/9313 